\title{
Metabolic syndrome in haemodialysis patients: prevalence, determinants and association to cardiovascular outcomes
}

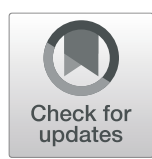

\author{
Arnaud Delautre ${ }^{1,2^{*}}$ (D) François Chantrel ${ }^{3,4}$, Yves Dimitrov ${ }^{4,5}$, Alexandre Klein ${ }^{4,6}$, Olivier Imhoff ${ }^{4,7}$, Clotilde Muller ${ }^{4,7}$, \\ Nicole Schauder ${ }^{8}$, Thierry Hannedouche ${ }^{1,2,4}$ and Thierry Krummel ${ }^{1 *}$
}

\begin{abstract}
Background: In the general population, metabolic syndrome (MetS) is predictive of major adverse cardiovascular events (MACE). Waist circumference (WC), a component of the MetS criteria, is linked to visceral obesity, which in turn is associated with MACE. However, in haemodialysis (HD) patients, the association between MetS, WC and MACE is unclear.

Methods: In a cross-sectional study of 1000 HD patients, we evaluated the prevalence and characterised the clinical predictors of MetS. The relationship between MetS and its components, alone or in combination, and MACE (coronary diseases, peripheral arteriopathy, stroke or cardiac failure), was studied using receiver operating characteristics (ROC) curves and logistic regression.

Results: A total of 753 patients were included between October 2011 and April 2013. The prevalence of MetS was $68.5 \%$. Waist circumference (> $88 \mathrm{~cm}$ in women, $102 \mathrm{~cm}$ in men) was the best predictor of MetS (sensitivity 80.2 ; specificity 82.3; AUC 0.80; $p<0.05)$. In multivariate analysis, MetS was associated with MACE (OR: 1.85; 95CI 1.24$2.75 ; p<0.01$ ), but not WC alone. There was a stronger association between the combination of abdominal obesity, hypertriglyceridaemia and low high-density lipoprotein cholesterol with MACE after exclusion of impaired fasting glucose and hypertension.

Conclusions: MetS is frequent and significantly associated with MACE in our haemodialysis cohort and probably in other European dialysis populations as well. In HD patients, a new simplified definition could be proposed in keeping with the concept of the "hypertriglyceridaemic waist".
\end{abstract}

Keywords: Metabolic syndrome, Haemodialysis, Waist circumference, Major cardiovascular events, Epidemiology

\section{Background}

Obesity is a significant risk factor for diabetes, high blood pressure and coronary heart disease in the general population and contributes to overall mortality worldwide. In dialysed patients, the relationship between body

\footnotetext{
*Correspondence: arnaud.delautre@chru-strasbourg.fr; thierry.krummel@chrustrasbourg.fr

${ }^{1}$ Service de Néphrologie et Dialyse, Hôpitaux Universitaires de Strasbourg, Strasbourg, France

Full list of author information is available at the end of the article
}

mass index (BMI) and mortality is more complex, with a $\mathrm{U}$ or $\mathrm{L}$ relationship $[1,2]$. The maximum risk of death is strongly associated with a low BMI while overweight and obesity are associated with either a modest increase in mortality rates or no increase at all [1-3]. These paradoxical results, coined "reverse epidemiology", have also been observed in other diseased populations including heart failure or cancer, particularly in observational short-term follow-up studies [4].

(c) The Author(s). 2020 Open Access This article is licensed under a Creative Commons Attribution 4.0 International License, which permits use, sharing, adaptation, distribution and reproduction in any medium or format, as long as you give appropriate credit to the original author(s) and the source, provide a link to the Creative Commons licence, and indicate if changes were made. The images or other third party material in this article are included in the article's Creative Commons licence, unless indicated otherwise in a credit line to the material. If material is not included in the article's Creative Commons licence and your intended use is not permitted by statutory regulation or exceeds the permitted use, you will need to obtain permission directly from the copyright holder. To view a copy of this licence, visit http://creativecommons.org/licenses/by/4.0/ The Creative Commons Public Domain Dedication waiver (http://creativecommons.org/publicdomain/zero/1.0/) applies to the data made available in this article, unless otherwise stated in a credit line to the data. 
BMI is a global parameter that does not dissociate abdominal adiposity (visceral white fat) which has deleterious metabolic effects, from muscle mass and brown fat which are protective factors [5]. Indeed, in the general population, several studies, notably the INTERHEART study, found a closer relationship between waist circumference (WC), which refers to abdominal obesity, and cardiovascular mortality, comparatively to BMI. After adjustment for diabetes and high blood pressure, BMI no longer had a predictive value on cardiovascular mortality [6]. Similarly, in the NHANES III study, WC was an independent predictor of cardiovascular mortality [7]. In another study, it was also the single parameter explaining the relationship between obesity and cardiovascular morbidity [8].

In the general population, studies have found that the presence of the metabolic syndrome (MetS), defined by the combination of abdominal fatness, lipid abnormalities, hyperglycaemia and hypertension, was a better indicator of cardiovascular death than obesity alone [9]. Occasionally, MetS has been considered as a more robust cardiovascular risk factor than diabetes alone $[10,11]$. MetS and diabetes were firmly associated, both in the general population [13] and dialysis patients. However, in diabetic patients, the metabolic syndrome is further associated with an increased cardiovascular risk [12]. The risk of post-transplant diabetes was also found to be predicted by pretransplantation MetS [14].

In the haemodialysis population, there are only few data on the relationship between MetS and cardiovascular morbidity and mortality. Most previous studies have focused on the prevalence of MetS. Still, these were small cohorts [15-26], originating from countries around the Mediterranean Sea or in Asia with a low baseline prevalence of MetS.

Besides, most studies in dialysis patients have been aimed at comparing the definition of metabolic syndrome according to various criteria (NCEP ATP III 2001, IDF 2005 and HMetS criteria 2009). In the present study, we used the latest 2009 classification (HMetS 2009), which is deemed as the most predictive of mortality in haemodialysis patients [15].

The primary objective of this study was to assess the prevalence of MetS in a large regional population of chronic haemodialysis patients, and to identify the predictors of MetS or its components, including waist circumference, either alone, or in combination.

The secondary objective was to establish the relationship between MetS and its components, alone or in association, with cardiovascular history and comorbidities and to compare the strength of this association with that of BMI and diabetes.

\section{Methods}

This observational cross-sectional study was conducted using data from the REIN registry (Nephrology Information and Epidemiological Network) nested in the Alsace region (Northeastern France) and was approved by the Comité Consultatif sur le Traitement de l'Information en matière de Recherche dans le domaine de la Santé (CCTIRS) and the Commission Nationale de l'Informatique et des Libertés (CNIL).

The following demographic data and clinical characteristics were collected in all patients treated by chronic haemodialysis in Alsace $(n=1000)$ between October 2011 and April 2013:

1. Age, gender

2. Weight, height, waist circumference (WC) and body mass index (BMI). WC was measured in each centre by the same operator according to the World Health Organisation (WHO) method [27]. The reference value in the analysis represents the average of 3 consecutive measurements.

3. Fasting serum HDL-cholesterol (HDL), LDLcholesterol (LDL), total cholesterol and triglycerides (TG)

4. History of high blood pressure or current antihypertensive treatment

5. Fasting plasma glucose, $\mathrm{HbA} 1 \mathrm{c}$

6. Diagnosis or history of diabetes

7. Smoking, current or past

8. Serum albumin

9. History of cardiovascular disease

- Stroke

- Congestive heart failure (CHF)

- Coronary heart disease (CHD):

- Myocardial infarction

- Presence of coronary stenosis

- Coronary revascularization with angioplasty or coronary artery bypass surgery

- Congestive heart failure (CHF).

- Peripheral arterial disease (PAD) (ordered stages 3 and 4)

10. Ongoing statin treatment

11. Dialysis vintage

All participating patients gave their informed consent to participate in the study. Data were extracted from computerised medical records Medware (Sined, Italy), shared by all dialysis centres of the region.

Metabolic syndrome (MetS) was defined according to the 2009 criteria (HMetS: « Harmonizing the Metabolic Syndrome ») [28]. The HMetS criteria suggest using either the 2005 or 2001 thresholds as a choice. We chose the highest, namely those of NCEP ATP III, recognised as adapted for European patients: 
1. Waist circumference (MsWC):

- $>102 \mathrm{~cm}$ in men

- $>88 \mathrm{~cm}$ in women

2. HDL-cholesterol (MsHDL)

- $<0.50 \mathrm{~g} / \mathrm{L}(<1.29 \mathrm{mmol} / \mathrm{L})$ in women

- $<0.40 \mathrm{~g} / \mathrm{L}(<1.03 \mathrm{mmol} / \mathrm{L})$ in men [29]

3. Triglycerides (MsTG) $\geq 1.5 \mathrm{~g} / \mathrm{L}(\geq 1.7 \mathrm{mmol} / \mathrm{L})$

4. Fasting blood glucose (MsGlc) $\geq 1.00 \mathrm{~g} / \mathrm{L}(\geq 5.6$ $\mathrm{mmol} / \mathrm{L}$ )

5. High blood pressure (MsHBP): Blood pressure values were not directly available and tend to exhibit cyclic variations in HD patients. Therefore, the "hypertension" criterion was defined by the declaration of a history of hypertension in the patient's record, or an ongoing antihypertensive treatment.

The presence of 3 criteria or more among the five was used to define the metabolic syndrome according to HMetS 2009 criteria [28].

Major adverse cardiovascular events (MACE) were defined according to the REIN registry criteria [30], by the presence of at least one of the following items in the patient's history:

- CHD (defined by a history of bypass surgery or angioplasty, or coronary artery disease documented by a stress test).

- Peripheral arterial disease (PAD) stage 3 or 4 (PAD 3-4), defined as stage 3: rest pain; stage 4: trophic disorders or amputation.

- Stroke with permanent sequelae (Stroke). Transient ischaemic attacks (TIAs), which have more uncertain diagnoses, were not included.

- Congestive heart failure (CHF).

\section{Statistical analyses}

Results are expressed as means \pm standard deviation for quantitative variables and compared with Student's $t$ test when following a normal distribution, and by the MannWhitney test for non-normal distributions. The normality of the distribution was tested graphically together with the Shapiro-Wilk test.

Qualitative variables are described as the frequency and percentage of each of the modalities and were compared using the Chi-square test.

The relationship between BMI and waist circumference was studied using the Bravais-Pearson correlation coefficient (normal distribution of the data).

Since diabetes may affect the presence of MetS and MACE, the analyses were stratified in both diabetic and non-diabetic patients.

The association between components of the metabolic syndrome and the full metabolic syndrome was studied using ROC curves and calculating sensitivities, specificities, positive and negative predictive values.

The association of waist circumference with the metabolic syndrome was also tested by logistic regression with adjustment for other parameters potentially associated with MetS including diabetes, haemoglobin A1c, BMI, smoking, age, gender, statin treatment and serum albumin.

Relationships between metabolic syndrome and history of cardiovascular events and cardiovascular comorbidities were studied in univariate analyses and then multivariate analysis. For the multivariate analysis, a logistic regression model was adjusted for diabetes, LDLcholesterol, smoking status, age, sex, BMI, statin use and serum albumin. The procedure was performed using a step-by-step backward selection (backward elimination). Parameters included in the multivariate analysis were those with a $P<0.20$ in the univariate analysis. Only serum albumin was "forced" into the multivariate analysis despite a $P>0.20$ given its acceptance as a significant prognostic factor in the dialysis population. Univariate and multivariate analyses were also repeated for studying the relationship between risk factors and coronary heart disease, PAD stage $3-4$, stroke and CHF individually.

The relationships between MACE and the various associations of the MetS criterion, but always including MsWC, were studied using the same univariate and multivariate models as described above.

The nonparametric Wilcoxon test was used to study the number of cardiovascular events between groups (abnormal distribution of the data).

All statistical analyses were performed with the STATA v14 software (Statacorp LLC, Texas, USA).

\section{Results}

The results were analysed for 753 patients $(75.3 \%$ of the initial cohort) for whom all data were available.

The missing data mostly consisted of WC measurements, not available in 128 patients (refusal of patients to participate or impossibility of standing upright for the measure), absence of fasting HDL and TG in 119 patients and absence of fasting blood glucose in 166 patients (Fig. 1).

For the 753 studied patients, the main demographic data are summarised in Table 1 and the presumed nephropathies leading to end-stage kidney disease in Table 2. There were 436 men (58\%) and 317 women (42\%). Mean patient age was $65.5 \pm 0.5$ years $(65 \pm 0.7$ years for men, $66.5 \pm 0.7$ years for women) while body mass index was $27.2 \pm 0.2 \mathrm{~kg} / \mathrm{m}^{2}$ for the whole population and $26.7 \pm 0.3 \mathrm{~kg} / \mathrm{m}^{2}$ and $27.9 \pm 0.4 \mathrm{~kg} / \mathrm{m}^{2}$ for men and women, respectively. Low BMIs $(<20 \mathrm{~kg} / \mathrm{m} 2)$ were observed in $8.5 \%$ of the cohort. Prevalence of overweight 


\section{Haemodialysis patients from Alsace}

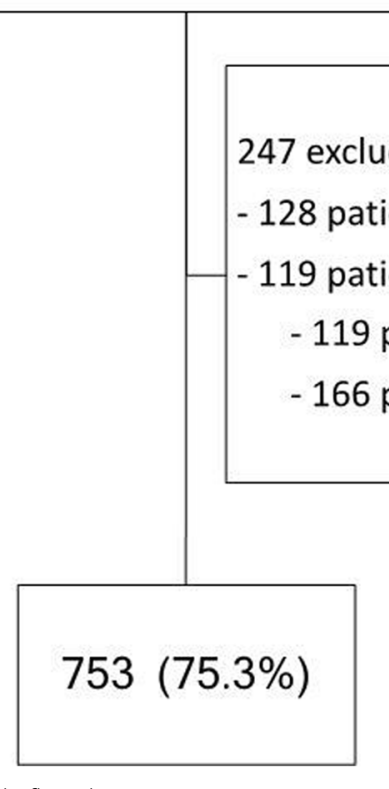

Fig. 1 Study flowchart

(BMI 25-29.99) and obesity $(\mathrm{BMI} \geq 30)$ was 32.1 and $28.3 \%$, respectively.

1) Prevalence and determinants of the metabolic syndrome

Of the 753 patients, $68.5 \%$ were diagnosed with MetS according to the HMetS definition, with a prevalence of $64.7 \%$ in men and $73.8 \%$ in women $(p<0.01)$.

A total of 456 patients $(60.6 \%)$ had MsWC. The mean WC was $102.0 \pm 0.6 \mathrm{~cm}$. The MsWC criterion was present in $48.8 \%$ of men and $76.7 \%$ of women, with an MsWC of $103.1 \pm 0.7 \mathrm{~cm}$ and $100.5 \pm 0.9 \mathrm{~cm}$, respectively.

In obese or overweight patients, 96.7 and $74.4 \%$ had MsWC, respectively. The relationship between BMI and WC was highly significant $(r=0.84 p<0.001)$, and equally observed in both genders.

Among the 753 patients, 343 (45.6\%) were diabetic, of whom $28.3 \%$ were obese and $19.4 \%$ overweight. In people with diabetes, WC was $109 \pm 0.8 \mathrm{~cm}$ and the MsWC criterion was found in $77 \%$ of these patients $(n=$ 264). The difference in WC between diabetic and nondiabetic patients $(96 \pm 0.7 \mathrm{~cm})$ was highly significant $(p<0.001)$.

In the whole cohort, the prevalence of the other MetS criteria, namely MsHDL, MsTG, MsGlc and MsHBP was $50.3,51.5,72$ and $87.3 \%$, respectively.
The association between the MetS components and MetS is shown in Table 3. There were 414 patients (55\%) with both MsWC and MetS while $80 \%$ of patients with MetS had MsWC (sensitivity: 80\%), including 197 (70\%) and 217 (93\%) in men and women, respectively. In patients without MetS, 195 did not have MsWC (specificity: $82 \%)$. The prevalence of MetS in patients with MsWC was 90.8\%.

ROC curves were plotted to study the relationship between the various individual parameters and MetS, from which areas under the curve (AUC) were calculated (Fig. 2). The following findings, in descending order, were observed:

\section{For both genders}

WC (0.80), TG (0.797), HDL (0.77), Glc (0.70). With $\mathrm{WC}=\mathrm{TG}(p=0.5)$, WC \& TG $>$ HDL $(p<0.05)$, HDL $>$ Glc $(p<0.05)$.

\section{In men}

HDL (0.83), WC (0.8012), TG (0.8011), Glc (0.68). With HDL $>$ WC $(p<0.05)$, WC $=$ TG $(p=0.5)$, WC \& TG > Glc $(p<0.05)$.

\section{In women}

WC (0.84), TG (0.79), HDL (0.76), Glc (0.74). With $\mathrm{WC}=\mathrm{TG}(p=0.5)$, WC \& TG $>$ HDL $(p<0.05), \mathrm{HDL}>$ Glc $(p<0.05)$. 
Table 1 Main clinical features and distribution of the components of the metabolic syndrome in the 753 haemodialysis patients with or without metabolic syndrome

\begin{tabular}{|c|c|c|c|}
\hline & MetS+ & MetS- & $p$ \\
\hline Prevalence. n (\%) & $516(68.5)$ & $237(31.5)$ & \\
\hline \multicolumn{4}{|l|}{ Gender, n (\%) } \\
\hline men & $282(54.7)$ & $154(65.0)$ & $<0.01$ \\
\hline women & $234(45.3)$ & $83(35)$ & \\
\hline Age, years & $66.6 \pm 0.5$ & $63.3 \pm 1.1$ & $<0.01$ \\
\hline Body height, cm & $165.2 \pm 0.4$ & $165.7 \pm 0.6$ & 0.50 \\
\hline Weight, kg & $79.2 \pm 0.8$ & $64.4 \pm 0.9$ & $<0.01$ \\
\hline Dialysis vintage, years & $5.0 \pm 0.2$ & $6.4 \pm 0.5$ & $<0.01$ \\
\hline Serum albumin, $\mathrm{g} / \mathrm{L}$ & $37.7 \pm 0.2$ & $37.9 \pm 0.3$ & 0.60 \\
\hline $\mathrm{BMl}, \mathrm{kg} / \mathrm{m} 2$ & $29.0 \pm 0.3$ & $23.4 \pm 0.3$ & $<0.01$ \\
\hline $\mathrm{BMI}>30, \mathrm{n}(\%)$ & $195(38)$ & $12(8)$ & $<0.01$ \\
\hline BMI 25-30, n (\%) & $184(36)$ & $50(21)$ & $<0.01$ \\
\hline $\mathrm{BMI}<20, \mathrm{n}(\%)$ & $13(3)$ & $51(22)$ & $<0.01$ \\
\hline Diabetes, n (\%) & $299(58)$ & $44(19)$ & $<0.01$ \\
\hline $\mathrm{HbA} 1 \mathrm{c}, \%$ & $6.7 \pm 0.2$ & $5.8 \pm 0.1$ & 0.01 \\
\hline Statin use, n (\%) & $329(64)$ & $121(51)$ & $<0.01$ \\
\hline Smoking, n (\%) & $228(54)$ & $103(53)$ & 0.80 \\
\hline$W C, \mathrm{~cm}$ & $107.4 \pm 0.6$ & $90.2 \pm 0.8$ & $<0.01$ \\
\hline Fasting glucose, $\mathrm{g} / \mathrm{L}$ & $1.3 \pm 0.03$ & $1.0 \pm 0.03$ & $<0.01$ \\
\hline $\mathrm{HDL}, \mathrm{mmol} / \mathrm{L}$ & $1.0 \pm 0.02$ & $1.4 \pm 0.03$ & $<0.01$ \\
\hline with statin, $\mathrm{mmol} / \mathrm{L}$ & $1.0 \pm 0.02$ & $1.4 \pm 0.03$ & $<0.01$ \\
\hline without statin, mmol/L & $1.1 \pm 0.02$ & $1.4 \pm 0.03$ & $<0.01$ \\
\hline $\mathrm{TG}, \mathrm{mmol} / \mathrm{L}$ & $2.4 \pm 0.06$ & $1.3 \pm 0.03$ & $<0.01$ \\
\hline with statin, mmol/L & $2.5 \pm 0.06$ & $1.3 \pm 0.03$ & $<0.01$ \\
\hline without statin, $\mathrm{mmol} / \mathrm{L}$ & $2.1 \pm 0.06$ & $1.3 \pm 0.03$ & $<0.01$ \\
\hline $\mathrm{LDL}, \mathrm{mmol} / \mathrm{L}$ & $3.1 \pm 0.1$ & $3.5 \pm 0.2$ & 0.03 \\
\hline with statin, mmol/L & $2.9 \pm 0.1$ & $2.9 \pm 0.1$ & 0.63 \\
\hline without statin, mmol/L & $3.5 \pm 0.1$ & $4.1 \pm 0.2$ & 0.06 \\
\hline MsWC, N (\%) & $414(80)$ & $42(18)$ & $<0.01$ \\
\hline men & $197(70)$ & $16(10)$ & $<0.01$ \\
\hline women & 217 (93) & $26(31)$ & $<0.01$ \\
\hline MsHBP, n (\%) & $483(74)$ & $174(26)$ & $<0.01$ \\
\hline MsHDL, n (\%) & $333(67)$ & $24(10)$ & $<0.01$ \\
\hline men & $187(66)$ & $13(8)$ & $<0.01$ \\
\hline women & $146(62)$ & $11(13)$ & $<0.01$ \\
\hline MsTG, n (\%) & $330(69)$ & $36(16)$ & $<0.01$ \\
\hline MsGlc, n (\%) & $423(85)$ & $93(43)$ & $<0.01$ \\
\hline
\end{tabular}

MetS+ Patients with metabolic syndrome; MetS- Patients without metabolic syndrome; WC Waist circumference; TG Triglycerides; MsWC Waist circumference $>102 \mathrm{~cm}$ in men or $>88 \mathrm{~cm}$ in women; MsHBP High blood pressure or antihypertensive drugs; $M s H D L ~ H D L$ cholesterol $<0.50 \mathrm{~g} / \mathrm{L}$ in women or $<0.40 \mathrm{~g} / \mathrm{L}$ in men; MsTG TG $\geq 1.5 \mathrm{~g} / \mathrm{L}$; MsGlc Fasting glucose $\geq 1.00 \mathrm{~g} / \mathrm{L}$.
Table 2 Distribution of presumed causes of kidney diseases in the 753 haemodialysis patients with or without metabolic syndrome

\begin{tabular}{lll}
\hline & MetS+ & MetS- \\
\hline Diabetes, n(\%) & $205(40)$ & $25(10)$ \\
Glomerulonephritis, n(\%) & $69(13)$ & $63(27)$ \\
Hypertension, n(\%) & $44(9)$ & $22(9)$ \\
Unknown, n(\%) & $48(9)$ & $25(10)$ \\
Polycystic, n(\%) & $33(6)$ & $18(8)$ \\
Pyelonephritis, n(\%) & $16(3)$ & $14(6)$ \\
Vascular, n(\%) & $7(1)$ & 0 \\
Autre, n(\%) & $94(18)$ & $70(30)$ \\
Total & $516(100)$ & $237(100)$ \\
\hline
\end{tabular}

MetS Metabolic syndrome

MetS was very highly prevalent in obese diabetics (97\%) and was found in "only" $48 \%$ of non-diabetic and non-obese patients.

The association of waist circumference with metabolic syndrome was tested with adjustment for diabetes, HbA1c, body mass index, tobacco use, age, sex, statin treatment and serum albumin. WC remained significantly associated with MetS after adjustment (OR 1.10. $p<0.001$; 95CI 1.07-1.13). Diabetes and male gender were also significantly associated with MetS with an OR of $3.51(2.0-6.23)$ and $0.38(0.20-0.72)$, respectively $(p<0.05$ for both).

BMI was conversely not significantly associated with MetS (OR 0.96; 95CI 0.87-1.07; $p=0.52$ ) (Table s1).

2) Metabolic syndrome and cardiovascular complications

Table 3 Association between MetS components and the presence or absence of MetS

\begin{tabular}{|c|c|c|c|c|c|c|}
\hline & & MsWC & MsHBP & MsHDL & MsTG & MsGlc \\
\hline \multirow[t]{4}{*}{ Total population } & Sensitivity & 80.23 & 93.6 & 69.81 & 68.89 & 84.6 \\
\hline & Specificity & 82.28 & 26.8 & 89.66 & 84.48 & 57.14 \\
\hline & PPV & 90.79 & 73.52 & 93.28 & 90.16 & 81.98 \\
\hline & NPV & 65.66 & 65.62 & 59.09 & 56.81 & 61.69 \\
\hline \multirow[t]{4}{*}{ Men } & Sensitivity & 69.86 & 93.97 & 71.37 & 70.61 & 84.56 \\
\hline & Specificity & 89.61 & 23.28 & 91.33 & 84.00 & 54.68 \\
\hline & PPV & 92.49 & 69.19 & 93.50 & 88.52 & 78.50 \\
\hline & NPV & 61.88 & 67.92 & 64.62 & 62.07 & 64.41 \\
\hline \multirow[t]{4}{*}{ Women } & Sensitivity & 92.74 & 93.16 & 67.91 & 66.82 & 84.65 \\
\hline & Specificity & 68.67 & 32.53 & 86.59 & 85.37 & 61.54 \\
\hline & PPV & 89.30 & 79.56 & 92.99 & 92.36 & 86.55 \\
\hline & NPV & 77.03 & 62.79 & 50.71 & 49.30 & 57.83 \\
\hline
\end{tabular}

MetS Metabolic syndrome. PPV Positive predictive value. NPV Negative predictive value. MsWC Waist circumference $>102 \mathrm{~cm}$ in men or $>88 \mathrm{~cm}$ in women. MsHBP High blood pressure or antihypertensive drugs. MsHDL HDL cholesterol $<0.50 \mathrm{~g} / \mathrm{L}$ in women or $<0.40 \mathrm{~g} / \mathrm{L}$ in men. MsTG TG $\geq 1.5 \mathrm{~g} / \mathrm{L}$. MsG/c Fasting glucose $\geq 1.00 \mathrm{~g} / \mathrm{L}$. 


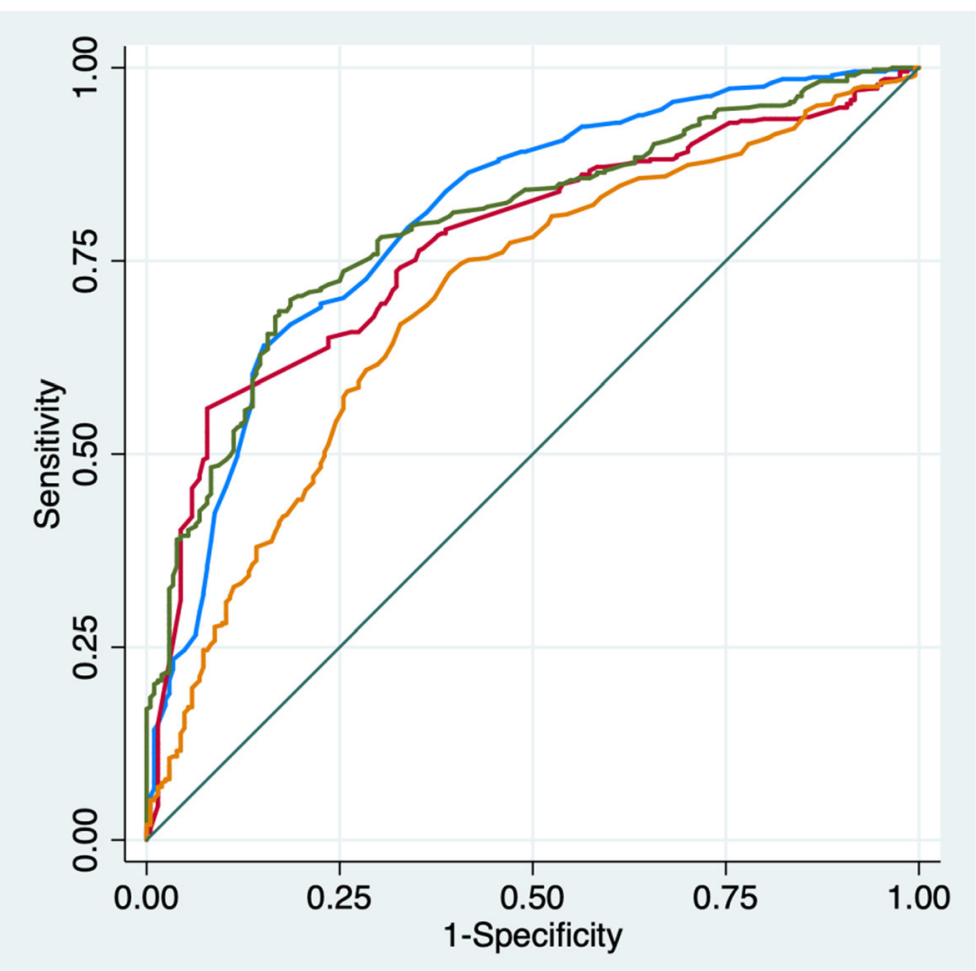

Fig. 2 ROC curves of the association between isolated MetS parameters and MetS. Legend: - Waist circumference, AUROC $=0.80$. $-\mathrm{HDL}$ cholesterol, $\mathrm{AUROC}=0.77$. $-\mathrm{TG}, \mathrm{AUROC}=0.797$. - Fasting glucose, $\mathrm{AUROC}=0.70$. AUROC: Area Under the ROC

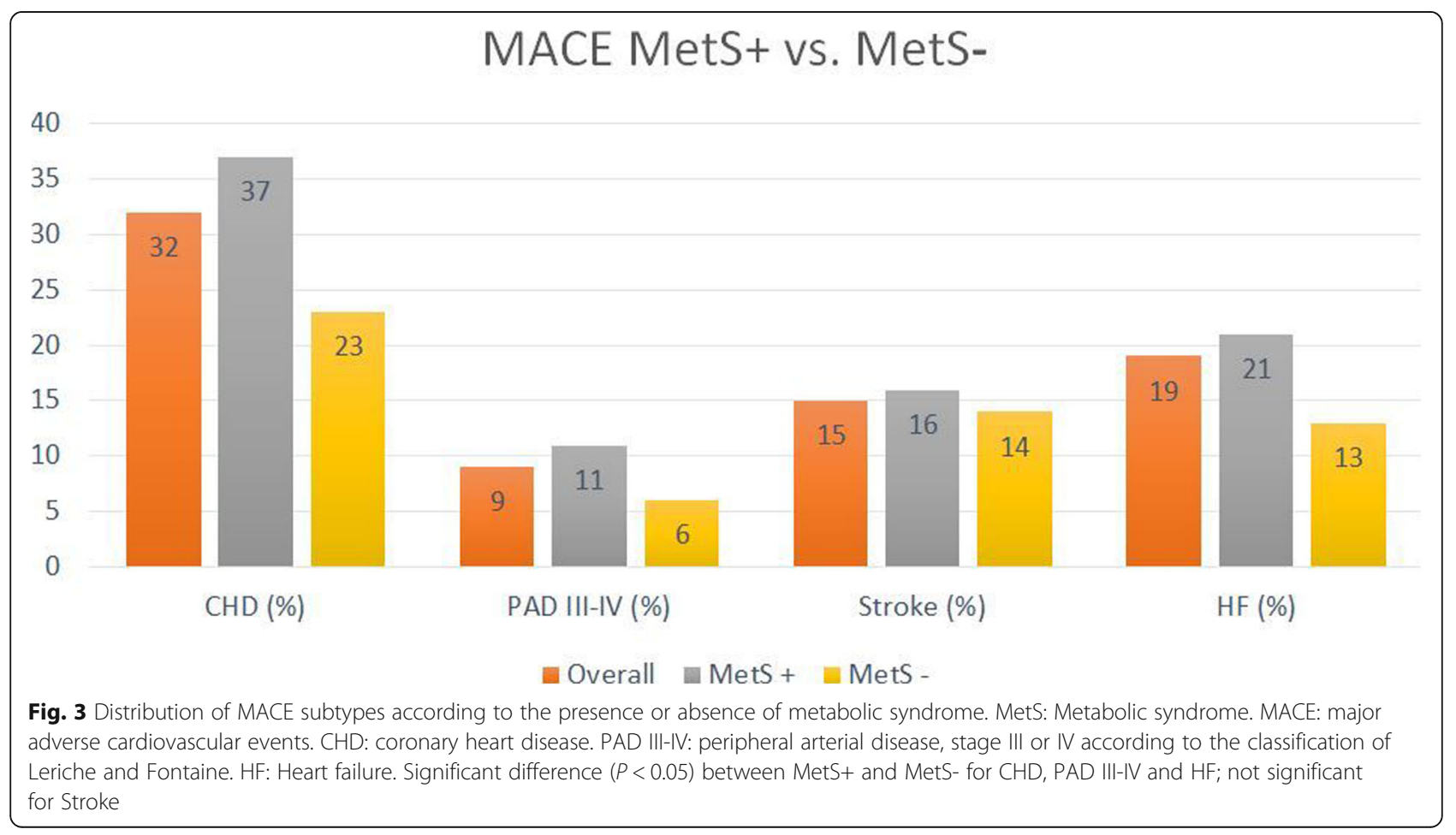


In the 753 patients, the distribution of MACE was: 244 patients $(32.4 \%)$ with $\mathrm{CHD}, 68$ patients (9\%) with stage 3 or 4 PAD, 114 patients (15.1\%) with stroke, and 140 patients $(18.6 \%)$ with congestive heart failure. In the whole cohort, 379 (50.3\%) had at least one cardiovascular complication, and 225 (29.9\%), 123 (16.3\%), 29 (3.8\%), and 2 (0.27\%) had one, two, three or four CV complications, respectively.

Among the 516 patients with MetS, 56\% exhibited at least one MACE compared to $38 \%$ for the group of patients without MetS. The individual subtypes of MACE based on the presence or absence of MetS are listed in Table s2 and Fig. 3.

The global MACE criterion was associated with MetS in univariate (OR 2.13; 95CI 1.56-2.92; $p<0.01$ ) as well as multivariate analysis (OR 1.85; 95CI 1.24-2.75; $p<0.01$ ) after adjustment for diabetes, LDL-cholesterol, smoking, age, sex, BMI, statin treatment and serum albumin (Table 4).

In univariate analysis, MetS was associated with coronary artery disease, congestive heart failure and PAD 3-4 with a OR of $2.0(1.4-2.8 ; p<0.001), 1.9(1.2-2.9 ; p=$ $0.005)$ and $1.9(1.0-3.5 ; p=0.04)$, respectively. In multivariate analysis, MetS was significantly associated with coronary artery disease (OR 1.92; 95CI 1.22-3.01; $p<0.01$ ) and heart failure (OR 2.35; 95CI 1.40-3.99; $p<0.01$ ), but not with PAD 3-4 or stroke (Table 5).

An association between MetS and cumulative MACE number $(p<0.001)$ was found with a mean of $0.84 \pm$ 0.04 MACE in patients with MetS compared to $0.55 \pm$ 0.5 in the group of patients without MetS.

\section{Components of the metabolic syndrome and MACE}

The association between the individual components of the metabolic syndrome and cardiovascular complications was also analysed.

There was a significant association between MsWC and the number of cardiovascular comorbidities with a

Table 4 Multivariate logistic regression of risk factors of overall major cardiovascular events

\begin{tabular}{llll}
\hline & OR & $p$ & $95 \% \mathrm{Cl}$ \\
\hline MetS & 1.85 & $<\mathbf{0 . 0 1}$ & $1.24-2.75$ \\
LDL $(\mathrm{g} / \mathrm{L})$ & 1.07 & 0.14 & $0.98-1.17$ \\
Diabetes & 1.58 & $\mathbf{0 . 0 2}$ & $1.08-2.32$ \\
Smoking & 1.78 & $<\mathbf{0 . 0 1}$ & $1.24-2.55$ \\
Age (year) & 1.03 & $<\mathbf{0 . 0 1}$ & $1.01-1.04$ \\
BMl (kg/m2) & 0.98 & 0.22 & $0.94-1.01$ \\
Statin use & 2.71 & $<\mathbf{0 . 0 1}$ & $1.87-3.93$ \\
Serum albumin $(\mathrm{g} / \mathrm{L})$ & 0.99 & 0.75 & $0.96-1.03$ \\
Male & 1.07 & 0.76 & $0.71-1.60$ \\
\hline
\end{tabular}

MetS Metabolic syndrome; LDL LDL cholesterol; BMI Body mass index. OR Odds ratio mean of $0.80 \pm 0.04 \mathrm{MACE}$ in the group with MsWC versus $0.67 \pm 0.05$ in the group without $\mathrm{MsWC}(P=$ 0.049 ). However, MsWC was only associated with coronary artery disease (OR 1.6; 95CI $1.2-2.2 ; p<0.05$ ) and PAD 3-4 (OR 1.8; 95CI 1.0-3.1; $p<0.05$ ) in both univariate and multivariate analysis (Table s3). In multivariate analysis, diabetes was significantly associated with cardiovascular events including coronary artery disease, PAD 3-4 and stroke (OR 1.76 [95CI 1.18-2.63], $p<0.001 ; 5.90$ [95CI 2.78-12.5], $p<0.001$; and 1.89 [95CI 1.14-3.14], $p<0.001$ ), but no significant association with heart failure.

The other components of the MetS taken individually were not associated with MACE in multivariate analysis, except for MsHDL (OR 1.56; 95CI 1.06-2.30; $p<0.02$ ).

However, in multivariate analysis, there was a significant relationship between MACE and a combination of MsWC + MsTG (OR 1.75; 95CI 1.09-2.81; $p=0.02$ ) and a combination of MsWC + MsTG + MsHDL (OR 2.11; 95CI 1.26-3.54; $p<0.01)$. In contrast, the combinations of 4 or 5 MetS criteria were not associated with MACE in multivariate analysis. These data are summarised in Table 6 and Figure s1.

Low serum albumin was associated with heart failure (OR 0.96; 95CI 0.92-0.99; $p=0.01$ ). Only MetS and low serum albumin were associated with heart failure in multivariate analysis.

In diabetic patients, MetS was not associated with MACE in multivariate analysis (OR 2.28; 95CI 0.995.24; $p<0.05)$ while in non-diabetic patients, MetS was associated with MACE in multivariate analysis (OR 1.88; 95CI 1.16-3.04; $p=0.01$ ).

\section{Discussion}

In the present study, the prevalence of the metabolic syndrome and its components, measured individually or in combination, was analysed in a comprehensive cohort of haemodialysed patients in Alsace. The analysis also assessed whether the metabolic syndrome or various combinations of its components were associated with cardiovascular history and comorbidities, as compared with BMI and diabetes.

Our multicentre study in 753 patients comprised a representative proportion of the regional haemodialysis population $(75 \%)$. Of note, half of the non-included patients were excluded because WC measurements were deemed unfeasible due to the inability to stand up. To our knowledge, the present patient population represents the largest studied cohort of patients treated on maintenance haemodialysis and dealing with MetS.

Irrespective of the criteria used, a high prevalence of MetS was observed, present in more than half of the patients. Abdominal obesity (increased waist circumference) was also prevalent, particularly in women. These 
Table 5 Multivariate logistic regression of risk factors according to specific major cardiovascular event

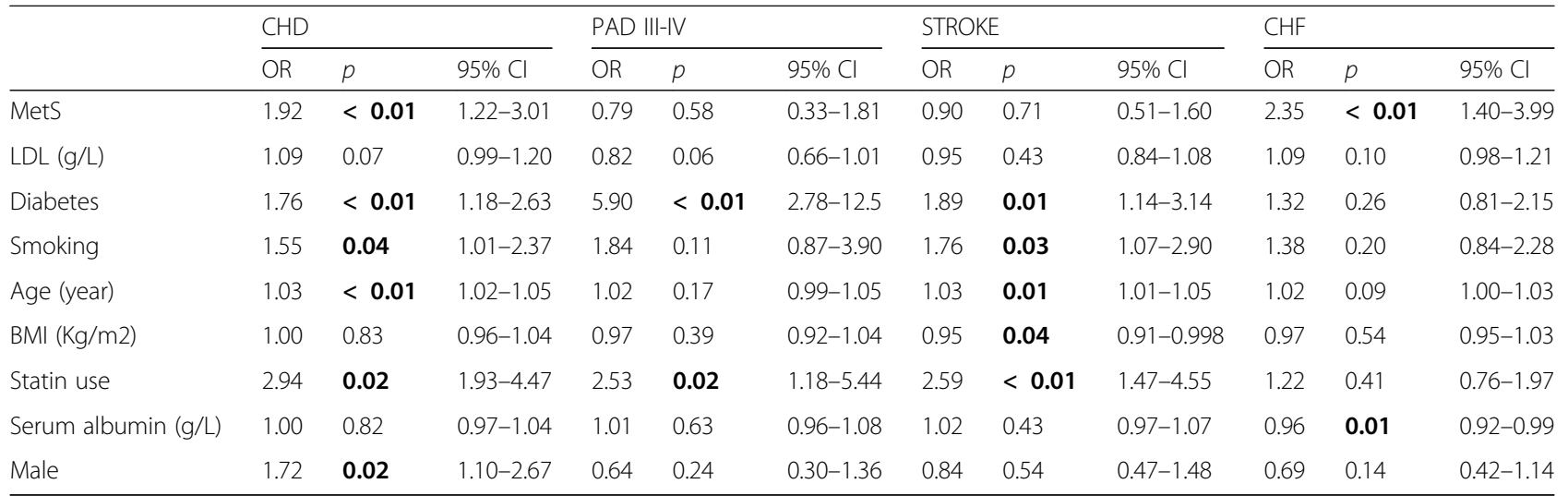

MetS Metabolic syndrome; LDL: LDL cholesterol; BMI Body mass index; CHD Coronary heart disease. PAD 3-4 Stage III or IV peripheral arterial disease; CHF Congestive heart failure

Table 6 Cumulative effects of metabolic syndrome components in predicting MACE in univariate and multivariate analysis. Multivariate analysis adjusted for diabetes, LDL-cholesterol, smoking, age, sex, BMI, statin use and serum albumin

\begin{tabular}{|c|c|c|c|c|c|c|c|}
\hline & \multirow[b]{2}{*}{ N/Nt(\%) } & \multicolumn{3}{|c|}{ Univariate analysis } & \multicolumn{3}{|c|}{ Multivariate analysis } \\
\hline & & $\overline{\mathrm{OR}}$ & $p$ & $95 \% \mathrm{Cl}$ & OR & $p$ & $95 \% \mathrm{Cl}$ \\
\hline \multicolumn{8}{|l|}{1 criterion } \\
\hline MsWC & 456/753 (60.6) & 1.32 & 0.063 & $0.99-1.77$ & 1.31 & 0.3 & $0.79-2.18$ \\
\hline MsTG & $366 / 711(51.5)$ & 1.46 & 0.01 & $1.09-1.96$ & 1.17 & 0.46 & $0.78-1.75$ \\
\hline MsHDL & $357 / 709$ (50.4) & 1.77 & $<0.01$ & $1.32-2.39$ & 1.56 & 0.02 & $1.06-2.30$ \\
\hline MsHBP & 657/753 (87.3) & 1.82 & $<0.01$ & $1.17-2.82$ & 1.76 & 0.049 & $1.0-3.10$ \\
\hline MsGlc & $516 / 717(72.0)$ & 2.67 & $<0.01$ & $1.90-3.75$ & 1.25 & 0.42 & $0.73-2.16$ \\
\hline \multicolumn{8}{|l|}{2 criteria } \\
\hline MsWC + MsTG & 254/720 (35.3) & 1.73 & $<0.01$ & $1.27-2.36$ & 1.75 & 0.02 & $1.09-2.81$ \\
\hline MsWC + MsHDL & 247/714 (34.6) & 1.71 & $<0.01$ & $1.25-2.33$ & 1.53 & 0.079 & $0.95-2.46$ \\
\hline MsWC + MsHBP & 413/753 (54.85) & 1.38 & 0.03 & $1.04-1.85$ & 1.48 & 0.093 & $0.94-2.32$ \\
\hline$M s W C+M s G l c$ & $361 / 741$ (48.7) & 1.83 & $<0.01$ & $1.37-2.45$ & 1.4 & 0.2 & $0.84-2.34$ \\
\hline \multicolumn{8}{|l|}{3 criteria } \\
\hline MsWC + MsTG + MsHDL & 169/716 (23.6) & 1.93 & $<0.01$ & $1.36-2.75$ & 2.11 & $<0.01$ & $1.26-3.54$ \\
\hline $\mathrm{MsWC}+\mathrm{MsHDL}+\mathrm{MsHBP}$ & 226/714 (31.7) & 1.78 & $<0.01$ & $1.29-2.45$ & 1.53 & 0.079 & $0.95-2.46$ \\
\hline$M s W C+M s H D L+M s G l c$ & 200/706 (28.3) & 2.05 & $<0.01$ & $1.47-2.86$ & 1.39 & 0.21 & $0.83-2.33$ \\
\hline $\mathrm{MsWC}+\mathrm{MsTG}+\mathrm{MsHBP}$ & $227 / 720(31.5)$ & 1.67 & $<0.01$ & $1.22-2.30$ & 1.39 & 0.15 & $0.89-2.16$ \\
\hline MsWC + MsTG + MsGlc & 211/712 (29.6) & 1.88 & $<0.01$ & $1.35-2.60$ & 1.28 & 0.34 & $0.77-2.12$ \\
\hline $\mathrm{MsWC}+\mathrm{MsGl}+\mathrm{MsHBP}$ & $327 / 743(44.0)$ & 1.89 & $<0.01$ & $1.41-2.53$ & 1.49 & 0.11 & $0.92-2.42$ \\
\hline \multicolumn{8}{|l|}{4 criteria } \\
\hline $\mathrm{MsWC}+\mathrm{MsGl}+\mathrm{MsHBP}+\mathrm{MsHDL}$ & 185/708 (26.1) & 2.16 & $<0.01$ & $1.53-3.05$ & 1.59 & 0.085 & $0.94-2.69$ \\
\hline $\mathrm{MsWC}+\mathrm{MsGlc}+\mathrm{MsHBP}+\mathrm{MsTG}$ & 189/714 (26.5) & 1.85 & $<0.01$ & $1.32-2.59$ & 1.2 & 0.5 & $0.72-2.00$ \\
\hline MsWC + MsGlc + MsHDL +MsTG & $145 / 712(20.4)$ & 1.96 & $<0.01$ & $1.35-2.85$ & 1.49 & 0.15 & $0.87-2.55$ \\
\hline $\mathrm{MsWC}+\mathrm{MsTG}+\mathrm{MsHBP}+\mathrm{MsHDL}$ & $152 / 716(21.2)$ & 2,00 & $<0.01$ & $1.39-2.90$ & 1.62 & 0.15 & $0.83-3.16$ \\
\hline \multicolumn{8}{|l|}{5 criteria } \\
\hline $\mathrm{MsWC}+\mathrm{MsGl}+\mathrm{MsHDL}+\mathrm{MsHBP}+\mathrm{MsTG}$ & $132 / 714(18.5)$ & 2.1 & $<0.01$ & $1.42-3.11$ & 1.58 & 0.11 & $0.9-2.8$ \\
\hline
\end{tabular}

MsWC Waist circumference $>102 \mathrm{~cm}$ in men or $>88 \mathrm{~cm}$ in women. MsHBP High blood pressure or antihypertensive drugs. MsHDL HDL cholesterol $<0.50 \mathrm{~g} / \mathrm{L}$ in women or $<0.40 \mathrm{~g} / \mathrm{L}$ in men. MsTG TG $\geq 1.5 \mathrm{~g} / \mathrm{L}$. MsG/c Fasting glucose $\geq 1.00 \mathrm{~g} / \mathrm{L}$. N/NT Number of patient with the criteria (MsWC, MSTG ...) / number of patients with available data. $O R$ Odds ratio 
data reflect the exceedingly high prevalence of diabetes and obesity in the general Alsatian population, which also explains diabetic nephropathy as the leading cause of end-stage renal failure in this region [30].

As expected, the relationships between obesity, diabetes, metabolic syndrome and high WC were strong. The metabolic syndrome is a complex entity involving several criteria for its definition, which has evolved over time. The present study used the criteria from the latest 2009 classification (HMetS 2009), which appear better suited to haemodialysis patients [15].

In our population, however, not all criteria had the same positive predictive value (PPV), as high WC, hypoHDLaemia and hypertriglyceridaemia were identified as the most specific. Unsurprisingly, an increased waist circumference, a marker of abdominal adiposity, was the single most potent factor associated with the metabolic syndrome in our cohort. In contrast, the "high blood pressure" criteria was less specific, likely because hypertension could result from a number of other causes in this dialysis population, including arterial stiffness and volume overload.

In this cross-sectional study, more than $50 \%$ of the patients exhibited at least one cardiovascular comorbidity, confirming the high burden of cardiovascular comorbidities in this population. However, in the present haemodialysis cohort, metabolic syndrome was strongly and independently associated with major cardiovascular events (coronary artery disease, peripheral arteriopathy, stroke or congestive heart failure), with this association persisting after adjustment for other cardiovascular risk factors, including diabetes and BMI. This association was even more robust with certain specific complications, namely coronary heart disease and congestive heart failure.

The strength of this association may, however, have been underestimated due to the cross-sectional nature of the analysis. Indeed, patients with MetS, who faced a higher risk, could have died prematurely after starting haemodialysis. Also, most of the patients in whom measurement of WC was impossible were more likely to be arteriopathic amputees or post-stroke hemiplegics.

As expected, diabetes was strongly associated with MACE in our cohort. In the general population, the relationship between diabetes and metabolic syndrome is strong [31]. In our cohort, however, the harmful effect of the metabolic syndrome persisted after adjustment for diabetes, demonstrating an independent prognostic impact. An analysis excluding diabetics further confirmed a significant association between the metabolic syndrome and MACE, confirming previous reports in the general population [32]. Nevertheless, in the subgroup analysis studying diabetic patients, there was no significant association between metabolic syndrome and MACE. A study with more power would probably prove a statistical link between MS and MACE in diabetic patients. In contrast, neither WC alone nor BMI were associated with cardiovascular history in multivariate analysis, thereby corroborating the data observed for BMI in the general population.

More surprisingly, statin treatment was associated with a 2.7-fold higher risk of cardiovascular complications. In our study, $60 \%$ of the patients were on statin treatment, including a majority of patients with MetS. Unlike LDLcholesterol, both HDL-cholesterol and triglycerides were ostensibly relatively uninfluenced by the statin treatment. Although randomised trials have failed to find any apparent benefits of statins in dialysis patients, a direct deleterious effect of statin is seemingly unlikely, this paradox likely reflecting an indication bias, since statins are prescribed preferentially in individuals at high risk or with a history of cardiovascular complications.

When analysing the cardiovascular events individually, the association with metabolic syndrome was significant for coronary heart disease and congestive heart failure but not for peripheral arteriopathy or stroke.

There are sparse data in the literature on the metabolic syndrome in dialysis patients, with data applicable to European populations being particularly scarce. Moreover, studies have yielded conflicting results. A prospective Tunisian study in 200 haemodialysis patients found an association between the metabolic syndrome and cardiovascular events [18]. Another Iranian retrospective study conducted in 300 haemodialysis patients showed an increased risk of coronary heart disease associated with the metabolic syndrome [19]. In contrast, the prospective study of Tsangalis et al. in 102 patients in Greece did not find an association between metabolic syndrome and MACE [20]. These authors suggested a survival bias in explaining these results since patients with metabolic syndrome could have been the first to die before inclusion in the study. In a prospective Chinese study, 157 patients exhibited no association between metabolic syndrome, CVD, and mortality [21]. In these two last studies, MetS was associated with a better nutritional status, a possible confounding factor. Of note, we did not find any difference in serum albumin levels in patients with or without the metabolic syndrome.

\section{Components and simplified MetS}

The current definition of the metabolic syndrome is somewhat complex as it involves parameters requiring a strictly fasting measurement (blood glucose, HDLcholesterol, triglycerides) or potentially associated with high variability (blood pressure). This may hinder the evaluation of MetS and raises the question of whether a simple measurement of waist circumference could provide similar prognostic information. 
In the present study, we found no significant association between high waist circumference (MsWC) and history of MACE, after adjustment for diabetes and BMI. These results are inconsistent with those of a prospective Italian study of the CREDIT registry (Calabria Registry of Dialysis and Transplantation) [16], which analysed the effect of waist circumference on global and cardiovascular mortality in 537 haemodialysis patients. In this south-Italian cohort, the prevalence of obesity and MsWC was much lower (12 and 39\% respectively) comparatively to the present cohort. While BMI was inversely correlated with mortality, the increase in WC was directly associated with an increased risk of overall and cardiovascular death. Moreover, after adjusting for other risk factors, a $10 \mathrm{~cm}$ higher $\mathrm{WC}$ was found to increase overall mortality and cardiovascular mortality by 26 and 38\%, respectively. Among all the other risk factors (cardiovascular history, age, duration of dialysis, haemoglobin, CRP), only the presence of diabetes increased the risk of overall (OR 2.73) and cardiovascular (OR 2.88) mortality. Unfortunately, this Italian study did not investigate the prognostic value of the full-blown metabolic syndrome on cardiovascular mortality.

In Japan, an association between directly CT-measured abdominal adiposity and cardiovascular disease, including stroke and coronary artery disease was observed in a small cohort of haemodialysis patients [17-26].

These findings, however, have not been consistent. In Taiwan, another prospective study in 91 patients found more cardiovascular events associated with the metabolic syndrome and increased WC [28], but no association with mortality [22]. These authors also found that abdominal obesity was correlated with PAD, independently of other factors related to MetS and inflammation [23].

BMI is a global parameter that does not dissociate abdominal adiposity (visceral white fat), which has harmful metabolic effects, from muscle mass and brown fat which, conversely, are protective factors [5, 33]. However, WC does not differentiate visceral adiposity which is more strongly associated with the risk of diabetes [34, 35], dyslipidaemia [36], hypertension [37] and CV complications $[5,38]$, from subcutaneous adiposity, which is potentially more metabolically benign. Visceral obesity can be better quantified using CT scans of L4-L5 [5, 24, $25,39]$, although this technique is poorly suitable for epidemiological studies. Some authors have proposed the concept of "hypertriglyceridaemic waist", associating a high waist circumference and an increase in serum triglycerides. This syndrome has even been found more strongly correlated with visceral obesity $[5,35]$ and cardiovascular diseases [40].

In the dialysis population, hypertension is prominent, although its association with cardiovascular morbidity and mortality remains controversial [41]. Since hypertension results from multiple mechanisms in haemodialysis patients [42], its integration into the definition of the metabolic syndrome is hence debatable.

Furthermore, our findings show that the combinations of MsWC + MsTG or MsWC + MsTG + MsHDL were those most significantly associated with cardiovascular complications. Indeed, the addition of a 4th or 5th parameter (either blood glucose or high blood pressure) did not yield any additional prognostic information. Our results thus support the use of the "hypertriglyceridaemic waist" as a marker of cardiovascular risk in haemodialysis patients.

Finally, only MetS and low serum albumin were associated with heart failure in multivariate analysis. In haemodialysis patients, low serum albumin is frequent and strongly associated with both cardiac and overall mortality [43-45]. Nevertheless, the significance of low serum albumin is multiple, including malnutrition but also chronic inflammation (IL6-dependent liver synthesis), as well as haemodilution related to volume overload.

The strengths of the study include the relatively large homogeneous cohort, representative of a contemporary European population. We also used a harmonised coding system nested in the regional REIN registry. Finally, the WC measurement was standardised across all participating centres.

Some limitations of the study should bear in mind. Although the cohort was representative, nearly $25 \%$ of the patients were excluded from the analysis, some of whom could have provided valuable information. Collection of MACE was performed retrospectively at the time of initiation of maintenance dialysis and inclusion in the REIN registry, and yearly thereafter. On the other hand, chart history during pre-dialysis follow-up was available in a vast majority of patients. Finally, this study was crosssectional with the inherent limitations due to surviving bias, and statistical associations cannot be ascertained as being causative.

\section{Conclusions}

In this cross-sectional study, the metabolic syndrome was common in our regional haemodialysis cohort and was strongly correlated with diabetes and obesity.

A robust relationship was found between the metabolic syndrome and cardiovascular history, a link which was not observed with BMI or waist circumference alone.

The "simplified metabolic syndrome", combining high $\mathrm{WC}$ + hyperTG + hypoHDL, was more effective in predicting CV history, a finding consistent with the concept of "hypertriglyceridaemic waist" and may suggest a 
redefinition of the syndrome, specific to haemodialysed populations.

A large prospective study in haemodialysis patients aimed at confirming the associations between metabolic syndrome and cardiovascular events and mortality, is currently underway.

\section{Supplementary information}

Supplementary information accompanies this paper at https://doi.org/10. 1186/s12882-020-02004-3.

Additional file 1 Table s1. Multivariate logistic regression of predictive risk factors of metabolic syndrome.

Additional file $\mathbf{2}$ Table s2. Distribution of MACE according to the presence or absence of metabolic syndrome (MetS+ vs. MetS-),

Additional file $\mathbf{3}$ Table s3. Association between individual MACE subtypes and MsWC in univariate analysis.

Additional file 4 Figure s1. Forest plots of MetS, MetS without BPH, and cumulative effect of components of the metabolic syndrome in predicting MACE in multivariate analysis.

\section{Abbreviations}

BMI: Body mass index; CHD: Coronary heart disease; CHF: Congestive heart failure; HD: Haemodialysis; HF: Heart failure; HMetS: Harmonizing the metabolic syndrome; LDL: LDL-cholesterol; MetS: Metabolic syndrome; MetS: Patients without metabolic syndrome; MsGlc: Fasting glucose $\geq 1.00 \mathrm{~g} / \mathrm{L}$; MsHBP: High blood pressure or antihypertensive drugs; MsHDL: HDL cholesterol $<0.50 \mathrm{~g} / \mathrm{L}$ in women or $<0.40 \mathrm{~g} / \mathrm{L}$ in men; MsTG: $\mathrm{TG} \geq 1.5 \mathrm{~g} / \mathrm{L}$; MsWC: Waist circumference $>102 \mathrm{~cm}$ in men or $>88 \mathrm{~cm}$ in women; MACE: Major adverse cardiovascular events; NPV: Negative predictive value; OR: Odds ratio; PAD: Peripheral arterial disease; PAD 3-4: Stage III or IV peripheral arterial disease; PPV: Positive predictive value; REIN registry : Nephrology information and epidemiological network registry;

TIAs: Transient ischaemic attacks; TG: Triglycerides; WC: Waist circumference; WHO: World health organisation

\section{Acknowledgements}

The authors wish to thank François Severac, MD, for statistical expertise, and Nadia Honoré, Sabrina Boime and the Clinical Research Technicians for coordinating the study at the centre level, the study-referent dialysis nurses and management for organising WC measurements and the collection of fasting blood samples.

\section{Authors' contributions}

Dr. AD for writing and statistical analysis. Pr TH for writing and proofreading. Dr. TK for statistical analysis. Dr. FC, Dr. YD, Dr. AK, Dr. OI, Dr. CM, Dr. NS for proofreading. The author (s) read and approved the final manuscript.

\section{Funding}

The study received a grant from the Agence de Biomedicine. The REIN registry is a nationwide registry supported and funded by the Agence de Biomedicine (1 Avenue du Stade de France. 93212 Saint-Denis, France).

\section{Availability of data and materials}

This study was conducted using data from the REIN registry (Nephrology Information and Epidemiological Network) in the Alsace region (Northeastern France).

\section{Ethics approval and consent to participate}

The study is nested in the REIN registry the data collection of which was approved by the CCTIRS and the CNIL. The study was validated (and funded) by the Scientific Council of the Agence de Biomedecine. All patients gave their written informed consent.

\section{Consent for publication}

Not Applicable.

\section{Competing interests}

None of the authors have any conflict of interest regarding the scope of this study.

\section{Author details}

${ }^{1}$ Service de Néphrologie et Dialyse, Hôpitaux Universitaires de Strasbourg,

Strasbourg, France. ${ }^{2}$ Faculté de Médecine, Université de Strasbourg,

Strasbourg, France. 'Service de Nephrologie, Centre Hospitalier Emile Muller de Mulhouse, Mulhouse, France. ${ }^{4}$ Association pour I'Utilisation du Rein Artificiel en Alsace (AURAL), Strasbourg, France. ${ }^{5}$ Service de Nephrologie, Centre Hospitalier Général de Haguenau, Haguenau, France. ${ }^{6}$ Service de Nephrologie, Centre Hospitalier Général de Colmar, Colmar, France. ${ }^{7}$ Service de Nephrologie, Clinique Sainte-Anne, Strasbourg, France. ${ }^{8}$ Observatoire Régional de la Santé d'Alsace (ORSAL), Strasbourg, France.

Received: 26 December 2019 Accepted: 3 August 2020

Published online: 13 August 2020

\section{References}

1. Kalantar-Zadeh K, Abbott KC, Salahudeen AK, Kilpatrick RD, Horwich TB. Survival advantages of obesity in dialysis patients. Am J Clin Nutr. 2005; 81(3):543-54

2. Lassalle M, Fezeu LK, Couchoud C, Hannedouche T, Massy ZA, Czernichow S. Obesity and access to kidney transplantation in patients starting dialysis: a prospective cohort study. PLoS One. 2017;12(5):e0176616.

3. Kalantar-Zadeh K, Rhee CM, Chou J, et al. The obesity paradox in kidney disease: how to reconcile it with obesity management. Kidney Int Rep. 2017;2(2):271-81.

4. Ahmadi SF, Streja E, Zahmatkesh G, et al. Reverse epidemiology of traditional cardiovascular risk factors in the geriatric population. J Am Med Dir Assoc. 2015;16(11):933-9.

5. Lemieux I, Poirier P, Bergeron J, et al. Hypertriglyceridemic waist: a useful screening phenotype in preventive cardiology? Can J Cardiol. 2007;25:28-31.

6. Yusuf $\mathrm{S}$, Hawken $\mathrm{S}$, Ounpuu $\mathrm{S}$, et al. Obesity and the risk of myocardial infarction in 27.000 participants from 52 countries: a case-control study. Lancet. 2005:366(9497):1640-9.

7. Sahakyan KR, Somers VK, Rodriguez JP, Jensen MD, Roger- VL, Singh P. Normal weight central obesity. Implications for total and cardiovascular mortality. Ann Intern Med. 2015;163(11):827-35.

8. Janssen I, Katzmarzyk PT, Ross R. Waist circumference and not body mass index explains obesity- related health risk 1-3. Am J Clin Nutr. 2004;79:379-84

9. Ärnlöv J, Ingelsson E, Sundström J, Lind L. Impact of body mass index and the metabolic syndrome on the risk of cardiovascular disease and death in middle-aged men. Circulation. 2010;121(2):230-6.

10. Yusuf $S$, Hawken $S$, Ounpuu $S$, et al. Effect of potentially modifiable risk factors associated with myocardial infarction in 52 countries (the INTERHEA RT study): case-control study. Lancet. 2004;364(9438):937-52.

11. Alexander CM, Landsman PB, Teutsch SM, Haffner SM. NCEP-defined metabolic syndrome, diabetes, and prevalence of coronary heart disease among NHANES III participants age 50 years and older. Diabetes. 2003;52(5): 1210-4.

12. Song $\mathrm{SH}$, Hardisty CA. Diagnosing metabolic syndrome in type 2 diabetes: does it matter? QJM. 2008;101(6):487-91.

13. Tenenbaum A, Fisman EZ. "The metabolic syndrome... is dead": These reports are an exaggeration. Cardiovasc Diabetol. 2011;10(1):11.

14. Bonet J, Martinez-Castelao A, Bayes B. Metabolic syndrome in hemodialysis patients as a risk factor for new-onset diabetes mellitus after renal transplant: a prospective observational study. Diabetes Metab Syndr Obes. 2013:6:339-46.

15. Vogt BP, Souza PL, Minicucci MF, Martin LC, Barretti P, Caramori JT. Metabolic syndrome criteria as predictors of insulin resistance, inflammation and mortality in chronic hemodialysis patients. Metab Syndr Relat Disord. 2014;12(8):443-9.

16. Postorino M, Marino C, Tripepi G, Zoccali C. Abdominal obesity and allcause and cardiovascular mortality in end-stage renal disease. J Am Coll Cardiol. 2009:53(15):1265-72

17. Okamoto T, Morimoto S, Ikenoue T, Furumatsu Y, Ichihara A. Visceral fat level is an independent risk factor for cardiovascular mortality in hemodialysis patients. Am J Nephrol. 2014;39(2):122-9. 
18. Sioud Olfa BO, Zohra EA, Hanen M, et al. Prevalence of metabolic syndrome in hemodialysis patients. Follow-up for three years: relationship with cardiovascular disease development. Biochem Mol Biol J. 2018;04(01):1-5.

19. Jalalzadeh M, Mousavinasab N, Soloki M, Miri R, Ghadiani MH, Hadizadeh M. Association between metabolic syndrome and coronary heart disease in patients on hemodialysis. Nephrourol Mon. 2015;7(1):1-8.

20. Tsangalis G, Papaconstantinou S, Kosmadakis G, Valis D, Zerefos N Prevalence of the metabolic syndrome in hemodialysis. Int J Artif Organs. 2007;30(2):118-23.

21. Xie Q, Zhang AH, Chen SY, et al. Metabolic syndrome is associated with better nutritional status, but not with cardiovascular disease or all-cause mortality in patients on haemodialysis. Arch Cardiovasc Dis. 2012;105(4):211-7.

22. Wu CC, Liou HH, Su PF, et al. Abdominal obesity is the most significant metabolic syndrome component predictive of cardiovascular events in chronic hemodialysis patients. Nephrol Dial Transplant. 2011;26(11):3689-95.

23. Hung PH, Tsai HB, Lin CH, Hung KY. Abdominal obesity is associated with peripheral artery disease in hemodialysis patients. PLoS One. 2013;8(6):e67555.

24. Yurugi T, Morimoto $S$, Okamoto T, et al. Accumulation of visceral fat in maintenance hemodialysis patients. Clin Exp Nephrol. 2012;16(1):156-63.

25. Tokunaga K, Matsuzawa Y, Ishikawa K, Tarui S. A novel technique for the determination of body fat by computed tomography. Int J Obes. 1983;7(5):437-45.

26. Moriyama Y, Eriguchi R, Sato Y, Nakaya Y. Chronic hemodialysis patients with visceral obesity have a higher risk for cardiovascular events. Asia Pac J Clin Nutr. 2011;20(1):109-17.

27. Circumference W, Ratio W-H. Report of a WHO expert consultation. Geneva: World Health Organisation; 2008. p. 5-7.

28. Alberti KGMM, Eckel RH, Grundy SM, et al. Harmonizing the metabolic syndrome: a joint interim statement of the international diabetes federation task force on epidemiology and prevention; national heart, lung, and blood institute; American heart association; world heart federation; international atherosclerosis society; and International Association for the Study of obesity. Circulation. 2009;120(16):1640-5.

29. Prasad GR. Metabolic syndrome and chronic kidney disease: current status and future directions. World J Nephrol. 2014;3(4):210.

30. Lassalle M, Monnet E, Ayav C, Hogan J, Moranne O, Couchoud C. 2017 annual report digest of the renal epidemiology information network (REIN) registry. Transpl Int. 2019;32(9):892-902.

31. Wilson PWF, D'Agostino RB, Parise $H$, Sullivan L, Meigs JB. Metabolic syndrome as a precursor of cardiovascular disease and type 2 diabetes mellitus. Circulation. 2005;112(20):3066-72.

32. Alexander CM, Landsman PB, Teutsch SM, Haffner SM. Prevalence of coronary heart disease among NHANES III participants age 50 years and older. Diabetes. 2003;52(5):1210-4.

33. Lemieux I. PoirierP, Bergeron J et al. Hypertriglyceridemic waist: a useful screening phenotype in preventive cardiology? Can J Cardiol. 2007;23(12):23B-31B.

34. Ross R, Aru J, Freeman J, Hudson R, Janssen I. Abdominal adiposity and insulin resistance in obese men. Am J Physiol Endocrinol Metab. 2002; 282(3):E657-563.

35. Fujioka S, Matsuzawa Y, Tokunaga K, Tarui S. Contribution of intraabdominal fat accumulation to the impairment of glucose and lipid metabolism in human obesity. Metabolism. 1987;36(1):54-9.

36. Despres JP, Moorjani S, Ferland M, et al. Adipose tissue distribution and plasma lipoprotein levels in obese women. Importance of intra-abdominal fat. Arteriosclerosis. 1989;9(2):203-10.

37. Hayashi T, Boyko EJ, Leonetti DL, et al. Visceral adiposity and the prevalence of hypertension in Japanese Americans. Circulation. 2003;108(14):1718-23.

38. Després JP, Lemieux I. Abdominal obesity and metabolic syndrome. Nature. 2006:444(7121):881-7.

39. Sjöström L, Kvist H, Cederblad A, Tylén U. Determination of total adipose tissue and body fat in women by computed tomography, 40K, and tritium. Am J Phys. 1986;250(6):E736-45.

40. Lemieux I, Pascot A, Couillard C, et al. Hypertriglyceridemic waist: a marker of the atherogenic metabolic triad (hyperinsulinemia; hyperapolipoprotein B; small, dense LDL) in men? Circulation. 2000;102(2):179-84.

41. Hannedouche T, Roth $H$, Krummel T, et al. Multiphasic effects of blood pressure on survival in hemodialysis patients. Kidney Int. 2016 Sep;90(3): 674-84.

42. Agarwal R. Hypervolemia is associated with increased mortality among hemodialysis patients. Hypertension. 2010;56(3):512-7.

43. Abbott KC, Glanton CW, Trespalacios FC, et al. Body mass index, dialysis modality, and survival: analysis of the United States renal data system
Dialysis morbidity and mortality wave II study. Kidney Int. 2004;65(2):597605.

44. Moreau-Gaudry X, Jean G, Genet L, et al. A simple protein-energy wasting score predicts survival in maintenance hemodialysis patients. J Ren Nutr. 2014;24(6):395-400.

45. Solbu MD, Mjøen G, Mark PB, et al. Predictors of atherosclerotic events in patients on haemodialysis: post hoc analyses from the AURORA study. Nephrol Dial Transplant. 2018;33(1):102-12.

\section{Publisher's Note}

Springer Nature remains neutral with regard to jurisdictional claims in published maps and institutional affiliations.

\section{Ready to submit your research? Choose BMC and benefit from:}

- fast, convenient online submission

- thorough peer review by experienced researchers in your field

- rapid publication on acceptance

- support for research data, including large and complex data types

- gold Open Access which fosters wider collaboration and increased citations

- maximum visibility for your research: over $100 \mathrm{M}$ website views per year

At BMC, research is always in progress.

Learn more biomedcentral.com/submissions 